\title{
Recommendations to improve imaging and analysis of brain lesion load and atrophy in longitudinal studies of multiple sclerosis
}

\author{
H. Vrenken • M. Jenkinson • M. A. Horsfield • M. Battaglini • R. A. van Schijndel • \\ E. Rostrup · J. J. G. Geurts · E. Fisher • A. Zijdenbos · J. Ashburner · \\ D. H. Miller - M. Filippi - F. Fazekas $\cdot$ M. Rovaris $\cdot$ A. Rovira $\cdot$ F. Barkhof $\cdot$ \\ N. de Stefano $\cdot$ MAGNIMS Study Group
}

Received: 10 November 2012/Accepted: 12 November 2012/Published online: 21 December 2012

(C) The Author(s) 2012. This article is published with open access at Springerlink.com

\begin{abstract}
Focal lesions and brain atrophy are the most extensively studied aspects of multiple sclerosis (MS), but the image acquisition and analysis techniques used can be further improved, especially those for studying withinpatient changes of lesion load and atrophy longitudinally. Improved accuracy and sensitivity will reduce the numbers of patients required to detect a given treatment effect in a trial, and ultimately, will allow reliable characterization of individual patients for personalized treatment. Based on open issues in the field of MS research, and the current
\end{abstract}

The members of MAGNIMS Study Group Steering Committee are A. Rovira, N. de Stefano, X. Montalban, F. Barkhof, C. Enzinger, M. Filippi, J. Frederiksen, L. Kappos, O. Ciccarelli, J. Palace, H. Vrenken, M.A. Rocca, T. Yousry.

H. Vrenken $(\bowtie) \cdot$ F. Barkhof

Department of Radiology, VU University Medical Center,

Amsterdam, The Netherlands

e-mail: h.vrenken@vumc.nl

H. Vrenken

Department of Physics and Medical Technology,

VU University Medical Center, Amsterdam, The Netherlands

M. Jenkinson

Oxford Centre for Functional Magnetic Resonance Imaging of the Brain, Oxford University, Oxford, UK

M. A. Horsfield

Department of Cardiovascular Sciences, University of Leicester, Leicester Royal Infirmary, Leicester, UK

M. Battaglini · N. de Stefano

Department of Neurological and Behavioral Sciences, University of Siena, Siena, Italy

R. A. van Schijndel

Image Analysis Center and Department of Radiology,

VU University Medical Center, Amsterdam, The Netherlands state of the art in magnetic resonance image analysis methods for assessing brain lesion load and atrophy, this paper makes recommendations to improve these measures for longitudinal studies of MS. Briefly, they are (1) images should be acquired using 3D pulse sequences, with nearisotropic spatial resolution and multiple image contrasts to allow more comprehensive analyses of lesion load and atrophy, across timepoints. Image artifacts need special attention given their effects on image analysis results. (2) Automated image segmentation methods integrating the assessment of lesion load and atrophy are desirable. (3) A standard dataset with benchmark results should be set up to facilitate development, calibration, and objective evaluation of image analysis methods for MS.

\section{E. Rostrup}

Centre for Healthy Aging, University of Copenhagen,

Glostrup, Denmark

\section{E. Rostrup}

Department of Diagnostics, Copenhagen University Hospital, Glostrup, Denmark

\section{J. J. G. Geurts}

Department of Anatomy and Neuroscience,

VU University Medical Center, Amsterdam, The Netherlands

\section{E. Fisher}

Department of Biomedical Engineering, Lerner Research

Institute, Cleveland Clinic, Cleveland, Ohio, USA

A. Zijdenbos

Biospective, Inc., Montreal, Quebec, Canada

J. Ashburner

Wellcome Trust Centre for Neuroimaging,

London, UK 
Keywords Magnetic resonance imaging .

Multiple sclerosis - Brain atrophy · White matter lesions · Image analysis

\section{Introduction}

Longitudinal magnetic resonance imaging (MRI) studies of focal brain lesions and brain atrophy play an important role in the study of multiple sclerosis (MS) in that they help to improve understanding of disease pathobiology [1] and its clinical and cognitive effects [2], and to investigate the effect of therapeutic strategies [3, 4]. MRI assessment of lesion burden and of volumetric changes in the brain cover both the focal and diffuse aspects of the underlying pathological processes and can be achieved using standard structural imaging pulse sequences. There are, however, several limitations in their application to the study of MS. In the brain, while white matter (WM) lesions can easily be detected using standard proton density (PD)/T2-weighted or FLAIR imaging, the detection of focal gray matter (GM) lesions by standard sequences is much less reliable [5], due to the different tissue composition and pathological substrates [1]. Regarding brain atrophy, volumetric measures are sensitive to MS-related changes due to neuroaxonal loss, gliosis, demyelination, and possibly remyelination, but are also influenced by many other biological factors such as the degree of edema and hydration status of the tissues (e.g., [6-8]). In addition, while image acquisition techniques have already been standardized to a large degree, the image analysis methods needed to obtain reliable measures are not yet standardized and yield variable results $[6,9]$. A recent review of the literature on correlative studies between MRI and histopathology in MS [10]

\section{H. Miller}

Department of Neuroinflammation, Queen Square Multiple Sclerosis Centre, UCL Institute of Neurology, London, UK

\section{Filippi}

Neuroimaging Research Unit, Institute of Experimental Neurology, Division of Neuroscience, San Raffaele Scientific Institute, Vita-Salute San Raffaele University, Milan, Italy

\section{F. Fazekas}

Department of Neurology, Medical University Graz, Graz, Austria

\section{Rovaris}

U.O. Riabilitazione Neuromotoria - Centro Sclerosi Multipla, IRCCS S. Maria Nascente - Fondazione Don Gnocchi, Milan, Italy

A. Rovira

MR Unit (Deparment of Radiology), Hospital Universitari

Vall d'Hebron, Barcelona, Spain recommended improvement of imaging specificity, highresolution image acquisition, and use of combination of imaging methods in longitudinal studies to gain a deeper understanding of the disease processes in MS.

Against this background, this paper focuses on what we consider desirable future developments in image acquisition and analysis for the longitudinal assessment of brain lesions and brain atrophy in MS. Previous guidelines formulated in 1998 [11] regarding quantitative MR image analysis in MS served as the background against which we examined the current state of the art to derive recommendations on the development and application of image analysis methods for optimal assessment of brain lesions and atrophy in MS. Other important issues, such as the assessment of lesions and atrophy in the spinal cord and optic nerve, as well as the heterogeneity of focal lesions and the patterns of tissue changes in the normal-appearing brain, were excluded from this paper since, with the possible exception of spinal cord abnormalities, all these investigations require more advanced imaging techniques, hampering large-scale implementation. In the following sections, for each topic we will first present a brief position statement followed by the reasoning behind it.

\section{Image acquisition}

\section{Position statement}

Image acquisition should use isotropic 3D pulse sequences with multiple image contrasts to improve and extend analyses of lesions and atrophy, across timepoints. Image artifacts need special attention since they have significant effects on image analysis results.

Reasoning

Increasing sensitivity for detecting and quantifying lesion and atrophy changes

While 2D (i.e., multislice) imaging has certainly proven its value in diagnosis and research, it is equally clear that with through-plane spatial resolutions on the order of $3 \mathrm{~mm}$, the sensitivity for detecting localized subtle tissue changes over time is limited. One problem that may occur is subject motion between the acquisition of two interleaved sets of slices, which can introduce substantial errors in lesion load measurement as recently described [12]. However, even when interleaved scanning-related problems are absent or can be resolved, 2D imaging introduces severe limitations. Not only do images with anisotropic spatial resolution contain no information about the spatial distribution of signal through the slice, but any co-registration between 
images from different timepoints will inevitably introduce interpolation artifacts. This is not only the case if repositioning is poor, but even when previously published repositioning guidelines [11] are followed. For example, in cases where there is substantial atrophy between scans, good repositioning of the MR slices cannot circumvent the need to deform the image in the through-plane direction to match the brain, which introduces interpolation errors. Conversely, 3D acquisition schemes offer the advantage of allowing improved through-plane spatial resolution. This leads to improved image registration, and also to smaller interpolation-induced resampling errors, compared to $2 \mathrm{D}$ images with thick slices. It is now feasible on most scanners to acquire 3D image datasets with (near) isotropic resolution in clinically acceptable scan times [13].

It is more practical to acquire images using $3 \mathrm{D}$ pulse sequences with $\mathrm{T} 1$ weighting than with $\mathrm{T} 2$ weighting, and T1-weighted 3D images have become the standard imaging method for the study of brain atrophy. The application of other contrasts with 3D imaging in practical scan times is being made possible through recent efforts combining 3 Tesla scanners, with phased-array receiver coils, parallel acquisition, and a variable flip angle scheme [14]. Such developments are crucial to the study of focal WM and GM lesions with resolution comparable to that achievable in T1weighted imaging. It is likely that the assessment of T2 lesion volume change, which is now an outcome measure in many clinical trials of new putative treatments for MS (e.g., $[15,16])$, will be more accurate when this improved spatial resolution is employed. In addition, several techniques can be tailored to the imaging of GM lesions (DIR, MP-RAGE, SPGR, PSIR) and can be implemented with 3D acquisition; these are being systematically evaluated [5, 13, 17-23].

Longitudinal group studies looking for subtle, localized changes in lesions would also benefit from high-resolution $3 \mathrm{D}$ acquisition. One example application is the group-level lesion probability mapping (LPM) approach, which several studies have used cross-sectionally [24-29], but few so far have used to investigate longitudinal changes [30, 31]. Another example is the assessment of localized lesional change in individual patients through subtraction imaging, which has been shown to be improved when using nearisotropic spatial-resolution 3D imaging compared to $2 \mathrm{D}$ imaging. Using 3D images, more active lesions were detected, and inter-rater reliability was greater than for 2D images [32]. It should be expected that detecting withinpatient changes and establishing relations at a group level would be more sensitive to minor differences if better spatial resolution is employed. Finally, the spatial, temporal, and possibly causal relations between brain lesions and brain atrophy [30,33,34], could be studied better if both the atrophy and the lesions were imaged with (near) isotropic spatial resolution of the order of $1 \mathrm{~mm}$.
It would clearly be advantageous to define a set of 3D imaging techniques with multiple contrasts that capture as many of the known aspects of brain changes in MS as possible in both the GM and WM, with optimized image contrast and good spatial resolution.

\section{Image artifacts}

Care must be taken to minimize image artifacts, which can have a large influence on the results from image analysis. Common artifacts include radiofrequency (RF) intensity non-uniformity, phase-encode ghosting, signal wrap, and geometric distortion due to gradient non-uniformity, and $\mathrm{B}_{0}$ inhomogeneity. A relevant review can be found here [35].

Radiofrequency non-uniformity, which results in slow spatial variations of signal intensity known as the bias field, is usually partially corrected during image acquisition, although the increasingly common use of coil arrays and very high field scanners has led to an increase in the prevalence, severity, and variability of bias field in most images. Image analysis methods should include a bias field correction if necessary, as is commonly done in tissue-type segmentation methods $[36,37]$ or using stand-alone correction methods such as N3 [38].

Phase-encode ghosting artifacts are due to a mismatch between the true phase of spins and the phase corresponding to their spatial position; the signal from these spins can then erroneously appear elsewhere in the image. They arise when a collection of spins moves between phase encoding and read-out, either through motion of the entire head (motion artifacts) or flow of blood or CSF (flow artifacts). Motion of the head should obviously be restricted as much as possible. Blood flow artifacts may yield substantial distortions of the signal along the phase-encode direction(s), hampering analysis of those regions. Flow artifacts due to blood may be diminished by using a presaturation slab on the neck to minimize signal emanating from the blood entering the head. Flow artifacts, which increase with gadolinium injection particularly at the posterior fossa, can also be minimized by reduction of phase shifts with flow compensation or gradient moment nulling, but with the penalty of increasing the echo time.

The wrap-around artifact is most frequently observed in a sagittally or coronally oriented $3 \mathrm{D}$ acquisition with tight planning of the volume in the anterior-posterior direction, where the nose wraps into the occipital lobe or cerebellum. However, for multiarray coils with parallel imaging, such artifacts can also occur within the brain. To allow wholebrain analyses, wrap-around artifacts, if unavoidable, should be kept outside brain voxels. For methods requiring information outside the brain, such as SPM-VBM, it may also be necessary for the subcutaneous fat and skin to be 
Table 1 Image artifacts and possible solutions

\begin{tabular}{|c|c|c|}
\hline Image artifact & Possible solution & $\begin{array}{l}\text { Limitation or } \\
\text { negative effect of } \\
\text { proposed solution }\end{array}$ \\
\hline $\begin{array}{l}\text { Spatial signal } \\
\text { intensity variation } \\
\text { due to RF field } \\
\text { inhomogeneity } \\
\text { (bias field) }\end{array}$ & $\begin{array}{l}\text { Measure RF bias } \\
\text { field at acquisition } \\
\text { Parallel transmission } \\
\text { becoming } \\
\text { available on latest } \\
\text { generation of } \\
\text { scanners } \\
\text { Correct using bias } \\
\text { field correction } \\
\text { algorithm }\end{array}$ & - \\
\hline Wrap-around artifacts & $\begin{array}{l}\text { Change read-out } \\
\text { direction and field- } \\
\text { of-view }\end{array}$ & $\begin{array}{l}\text { Probable increase in } \\
\text { acquisition duration }\end{array}$ \\
\hline $\begin{array}{l}\text { Ghosting artifacts } \\
\text { (motion) }\end{array}$ & $\begin{array}{l}\text { Make patient as } \\
\text { comfortable as } \\
\text { possible } \\
\text { Limit duration of } \\
\text { acquisition }\end{array}$ & $\begin{array}{l}\text { Too short an } \\
\text { acquisition will lead } \\
\text { to unacceptably low } \\
\text { signal-to-noise ratio }\end{array}$ \\
\hline $\begin{array}{l}\text { Ghosting artifacts } \\
\text { (blood and CSF } \\
\text { flow) }\end{array}$ & $\begin{array}{l}\text { Apply pre-saturation } \\
\text { slab on neck, or } \\
\text { perform flow- } \\
\text { compensated } \\
\text { acquisition }\end{array}$ & $\begin{array}{l}\text { Effects on acquisition } \\
\text { duration and } \\
\text { outcome measures } \\
\text { to be evaluated }\end{array}$ \\
\hline $\begin{array}{l}\text { Geometric distortion } \\
\text { due to gradient non- } \\
\text { uniformity }\end{array}$ & $\begin{array}{l}\text { Correct using } \\
\text { existing algorithms }\end{array}$ & $\begin{array}{l}\text { Additional } \\
\text { interpolation; for } \\
\text { SIENA analysis, } \\
\text { any negative effects } \\
\text { seem to be } \\
\text { outweighed by } \\
\text { benefits }\end{array}$ \\
\hline $\begin{array}{r}\mathrm{B}_{0} \text { inhomogeneity } \\
\text { (usually limited) }\end{array}$ & $\begin{array}{l}\text { Shorten TE and use } \\
\text { higher strength } \\
\text { imaging gradients } \\
\text { Apply post hoc } \\
\text { correction }\end{array}$ & $\begin{array}{l}\text { Not always possible; } \\
\text { can compromise } \\
\text { signal-to-noise ratic } \\
\text { Additional } \\
\text { measurement of B0 } \\
\text { required }\end{array}$ \\
\hline $\begin{array}{l}\text { Poor SNR and/or } \\
\text { tissue contrast }\end{array}$ & $\begin{array}{l}\text { Optimize pulse } \\
\text { sequence design } \\
\text { and parameter } \\
\text { values }\end{array}$ & $\begin{array}{l}\text { SNR increase at } \\
\text { unchanged } \\
\text { resolution may lead } \\
\text { to increased } \\
\text { acquisition } \\
\text { duration; trade-off } \\
\text { to be made }\end{array}$ \\
\hline
\end{tabular}

kept clear of such artifacts. This can be achieved by proper choice of the read-out direction and field-of-view, albeit probably sometimes at the cost of increased scan time.

Non-uniformity of imaging gradients gives rise to geometric distortion, due to a violation of the assumption of a linear relation between field strength and true spatial position. When uncorrected, this has been shown to substantially affect whole-brain and local atrophy rate measurement $[39,40]$. The correction to remove the geometric distortion [41] does come at the cost of an additional image interpolation step which affects all subsequent analyses (although some recent, so far unpublished work has been done to combine this with other interpolations into a single step), but even so, for some analysis software (e.g., SIENA [42]) the beneficial effects of removing the distortion are greater than the potential loss of accuracy due to this additional interpolation [40].

$\mathrm{B}_{0}$ inhomogeneities also give rise to geometric distortion, but additionally cause signal intensity loss due to more rapid dephasing. Signal loss can be minimized by using spin-echo pulse sequences with high receiver bandwidths (high gradient strength); by acquiring for each slice a pair of images with opposite polarity gradients; or by applying a post hoc correction based on direct measurement of a $B_{0}$ map. However, it is worth noting that $B_{0}$ inhomogeneities are not normally significant for the type of images under consideration here, except in very high field strength scanners or with pulse sequences with long gradient echo train lengths.

For completeness, we should also consider poor SNR and tissue contrast as obvious factors influencing poor image analysis outcomes. Optimizing SNR and tissue contrast through choice of field strength, pulse sequence design, and optimization of sequence parameters prior to initiation of a study is imperative. Table 1 lists these artifacts and possible solutions.

\section{Image analysis}

\section{Position statement}

Improved automated image segmentation is needed to overcome the limitations of existing methods. They should be directed toward providing an integrated assessment of lesions and atrophy.

\section{Reasoning}

\section{Standardizing lesions and atrophy measurement}

Volumetric quantification of the changes in lesion load and cerebral atrophy depends crucially on tissue-type segmentation, which is influenced by both acquisition- and disease-related factors. Focusing on the disease-related factors, several recent studies have shown that the extent of WM lesions can influence GM atrophy measurements because WM lesions have MR properties similar to those of GM [43-48]. An interesting approach that has been proposed to counter this problem is lesion inpainting, whereby signal intensities of lesion voxels are substituted with those observed in normal-appearing WM, prior to further analysis [43, 45, 48]. Although this appears to be a 
promising approach yielding seemingly improved atrophy measurements [44, 46], the effect of the correction may change with the lesion load and the specific algorithms used for correction and segmentation. For the FAST segmentation software from FSL [37], the choice of partial volume modeling algorithm utilized by the segmentation method was shown to exert a clear influence [43]. An obvious limitation of the lesion inpainting approach is that the lesion voxels still have to be identified and correctly segmented before new intensities can be assigned to them prior to GM segmentation.

Ideally, however, tissue segmentation methods for longitudinal studies of MS should tackle these issues automatically, and we recommend that this should be done by concurrently analyzing all tissue classes. Indeed, an attempt at integrated segmentation including both lesion and atrophy assessments for a single timepoint has already been reported [47, 49]. The inclusion of all timepoints available for a patient in a single segmentation process is another step that might improve quantification. Such concurrent analysis of multiple timepoints for one patient has been implemented in the CLADA software for longitudinal cortical atrophy measurement [50] and in the FreeSurfer software package for cortical thickness measurement and deep GM volumetry [51], while another paper demonstrated how difference images, obtained by subtracting coregistered images from two timepoints, may be used in the automated quantification of lesion volume change [52].

Development of this type of integrated analysis may take substantial amounts of time, and not all issues may be solvable. It would therefore be prudent to investigate alternative approaches; such approaches could be informed by a detailed analysis of the errors that occur when applying current methods to data already collected in longitudinal studies of MS. While the "holy grail" of a comprehensive segmentation method accessible by all researchers in the field should still be pursued, improvement of existing techniques may be a useful alternative approach.

\section{Most frequent sources of errors}

Errors in image analysis in MS studies can be grouped into two main categories: poor registration quality, and poor tissue segmentation. In many analyses, the final tissue segmentation is preceded by an algorithm to (approximately) find the intracranial cavity $[53,54]$; in that case, a third category is the incorrect inclusion of extracranial tissue in the final segmentation. Errors in each of these categories are often the result of one of a few main causes:

- pathological changes, such as severe atrophy or large WM lesion load;
- image acquisition-related factors, such as incomplete head coverage, inadequate spatial resolution (leading to substantial partial volume effects), poor tissue contrast, limited SNR, and artifacts;

- inherent limitations of the algorithm, possibly aggravated by image acquisition-related factors.

Beyond the obvious (partial) solutions of both optimizing the image acquisition for the desired analysis (e.g., using full-head coverage whenever possible), and optimizing the analysis algorithms, there are several additional steps that allow relatively easy correction or prevention of such errors, which give substantial improvements to the quality of the analyses. For group studies, registration errors due to the presence of severe pathology may be limited by using disease group-specific templates rather than standard healthy control templates, together with appropriate regularization of the registration [55]. However, when there are large pathological changes within a single patient, adequate non-linear matching between timepoints remains challenging. Errors in segmentation may be limited by using information from more than one image type, ideally in an integrated segmentation approach as recommended above. For both these issues, challenges remain, and solving both might be facilitated by the standardized test dataset discussed under recommendation (3).

Progress has recently been made in the initial segmentation of the intracranial cavity, often referred to as "brain extraction". Brain extraction is often imperfect, leaving tissue around the eyes and optic nerves, or removing part of the brain tissue, thus potentially introducing large errors in atrophy measurements by tools that rely on the brain extraction accuracy. A previous study showed that for $2 \mathrm{D}$ images, manual correction of the brain extraction used by SIENA (BET) increases sensitivity to disease effects in MS [56], but this solution is not feasible for high-resolution 3D images due to the high workload that would be generated. In this case, the brain extraction option settings should be optimized until the best compromise in brain extraction is obtained across all the images to be analyzed. However, a recent paper showed that a single combination of option settings yielded quantitatively very good results across a range of 3D T1-weighted image types in MS patients [57], obviating the need for further adjustment.

\section{Lesions}

Many lesion segmentation algorithms have been proposed, and a useful recent review is given in [58]. We restrict the scope here to fully automated methods and those that require minimal user intervention. The methods are based on several different principles such as intensity thresholding (e.g., [59, 60]), intensity gradient features (e.g., [61]), 
intensity histogram modeling of the expected tissue classes (e.g., [49, 62, 63]), identification of nearest neighbors (from a training data set) in a feature space (e.g., [64-66]), or fuzzy connectedness (e.g., [67, 68]), often using several of these in combination. In some cases spatial (anatomical) information is included in addition to intensities (e.g., [49, 67, 69]). Algorithmic approaches to segmentation optimization include methods such as Bayesian, expectation maximization, support vector machines (e.g., [70]), k-nearest neighbor majority voting (e.g., [64, 65], and artificial neural networks (e.g., [71].

Although promising results are often reported for images from a single scanner, performance on diverse datasets can be poor due to the different tissue contrasts that may be unknown to the algorithm. This can result in large fractions of both false-positives and -negatives; these misclassifications have proved to be a barrier to widespread adoption, especially in longitudinal studies if image quality varies over time and the level of these misclassifications is inconsistent. Incorporation of "domain knowledge", i.e., prior knowledge of the distribution of MS lesions in the brain, improves the segmentation of lesions [67], but, in our experience still does not deliver segmentations that are acceptable to researchers in the field. Because of this unreliability, practical lesion segmentation methods are generally not fully automated, and operator intervention is still needed at the level of individual lesions, usually by some form feature selection based on the local maximum intensity gradient, followed by contour following, e.g., [72-75]. Intra- and inter-observer reproducibilities of contouring are better than for manual outlining [76, 77], but the method is still labor-intensive. In order to be able to handle the large volumes of imaging data emanating from large therapeutic trials, it would seem appropriate to strive for further, if not complete, automation.

Regarding automated quantification of lesion load change, a recent review by Lladó et al. [78] highlights the state of the art and remaining challenges for application in a clinical or clinical trial setting. This review includes a table that clearly shows the lack of consistency in quantitative performance metrics used in the literature, clearly illustrating the need for standardized reporting methods. Lladó et al. classify methods for change quantification as intensity-based analysis, temporal analysis, and deformationbased analysis. An intensity-based approach to the detection of change in lesions over time could exploit a combination of registration and subtraction as used by Moraal et al. [32, $79,80]$. If an expert reviewer is available, the registrationsubtraction approach allows easy identification of change, provided that the changes between timepoints due to atrophy are not too large, or a registration method is used that can deal with the resulting brain shape deformations. It was shown for $2 \mathrm{D}$ images that the number of changing $\mathrm{T} 2$ lesions observed from the beginning to the end of a trial is statistically more powerful than the number of gadoliniumenhancing lesions from monthly scans [80]. Duan et al. [52] showed the feasibility of automatically quantifying these changes in lesions from the difference images.

The methods that Lladó et al. refer to as temporal methods typically handle image series with a large number of timepoints, which is an advantage over subtraction image analysis which can only handle two timepoints at once. The method proposed by Ait-Ali et al. [81] uses expectation maximization to first estimate non-lesion tissues and then adds lesions to the model. Gerig and colleagues [82] first perform segmentation of GM and WM, and then identify active lesions based on voxel mean and variance over the course of the timepoints. Although the method by Gerig et al. leaves room for improvement, most clearly regarding between-timepoint registration (assumed to be perfect) and the model for temporal signal evolution of MS lesions (assumed to be highly similar between lesions), it does present a feasible approach to the multipletimepoint analysis of lesions.

Deformation-based methods for lesion change quantification use the local volume change as calculated through deformable registration methods to quantify the lesion volume change. Two viable methods for lesion change quantification have been presented, i.e., that by Rey et al. [83], which is based on Thirion and Calmon [84], and that by Pieperhoff et al. [85], but both require additional modeling or operator intervention to indicate which are the lesion areas whose volume change should be quantified. The lesion segmentation problem therefore still needs to be solved in these approaches.

Three-dimensional imaging with isotropic resolution and multiple image contrasts can be expected to further increase the specificity with which change in lesions can be characterized, both in terms of their spatial location and for distinguishing and interrelating changes in different lesion types. For all these methods, there are several choices to be made on issues such as the type of registration, whether and how to include prior information on expected lesion and atrophy-related change, among others; these choices should be informed in part by comparing results against expert manual analysis.

\section{Atrophy}

Just as analysis of MS lesions in longitudinal studies is affected by concomitant atrophy, so too does atrophy quantification deteriorate when there are large changes in the lesion load. For example, large changes in atrophy or in lesion volumes may disrupt the accuracy of registration, which is used by many atrophy measurement methods [ 86,87$]$.

In normal aging and Alzheimer's disease (AD), Smith et al. compared two whole-brain atrophy measurement 
techniques, i.e., (brain) boundary shift integral (BSI [88]) and SIENA directly and showed that the methods gave very comparable results [89]. Sample size calculations in RRMS showed similar sample sizes were required for BSI and SIENA [90]. Using images with simulated atrophy in AD, Camara et al. [91] confirmed the good agreement between BSI and SIENA. More recently, Durand-Dubief et al. [6] selected seven methods for measuring whole-brain atrophy and assessed their reproducibility across different MRI platforms. This study on nine patients scanned on three occasions over 1 year, each time on two MRI scanners, showed that registration-based methods, i.e., where the registration is performed within-subject between timepoints, particularly an optimized BSI method using k-mean clustering (KNBSI) and Jacobian integration, gave the best agreement of whole-brain atrophy measures between the two different MRI scanners.

Also in MS, but focusing on local change instead, Battaglini et al. [92] performed a qualitative comparison between two different methods for measuring local changes in atrophy over time. By comparing longitudinal VBM (using FSL) and the voxelwise SIENA-R method directly, in the same longitudinal image set from MS patients who were scanned twice, with a 3-year interval between the two scans, they showed that the cortical regions in which significant atrophy was observed were roughly similar, but the extent was very different. This result was perhaps to be expected based on the different mechanisms of the two methods, with VBM quantifying local GM density and its change over time, while SIENA-R measures displacement of the local brain-non-brain boundary. Nevertheless, this study demonstrates the influence that choice of analysis method has on the results. Both this difference between SIENA-R and longitudinal VBM, and the superiority of (within-subject) registration-based techniques may be explained by the design of the methods: analysis methods that analyze within-subject change over time directly, by concurrently analyzing multiple timepoints, make use of the fact that intra-subject variability is generally smaller than inter-subject variability. These inherently longitudinal methods may therefore be better at quantifying this change than methods that treat each timepoint separately.

As indicated in the section on image acquisition, results are also influenced by the choice of imaging parameters, and so tissue contrast and spatial resolution should be optimized. Nevertheless, the CLADA method proposed by Nakamura et al. [50] did achieve both accurate measurement of cortical thickness, and reliable measurement of cortical thickness change, in low-resolution 2D images that are (still) typical for clinical trials. Accuracy may also differ between local atrophy measurement techniques, as shown quantitatively by the simulated $\mathrm{AD}$ atrophy study by Camara et al. [91]: deviations from ground truth atrophy differed between two Jacobian integration methods.
Moreover, the mean absolute deviation was up to $93 \%$ of the ground truth volume change for hippocampus, indicating the need for further method improvement. Partly simulated image data in which the true change is known, as used in their study, may also facilitate such developments in MS, especially when based on representative images from MS patients and made widely available as recommended below.

In healthy subjects with a mean age of 56.5 years, Takao et al. [93] investigated the effect of scanner performance on whole-brain and local volume change measurement. They showed that scanner drift and inter-scanner variability can produce large apparent volumetric changes in VBM (using SPM), including both increases and decreases. In contrast, a recent paper on MS demonstrated that, following a standardized imaging protocol and identical longitudinal VBM analysis methods, the differences between centers in the longitudinal VBM changes observed in MS patients are much smaller than the disease-related changes, indicating that pooling of data from different centers may be feasible for longitudinal VBM analysis in MS [94]. These scanner effects are important issues in most large-scale studies in MS, and this discrepancy merits further investigation.

\section{Available methods and proposed direction}

Table 2 lists the currently available methods for lesion load and atrophy measurement. The list is restricted to those methods that are available for installing locally on the researchers' own systems (not necessarily without charge). The merits and limitations of each method are briefly indicated. It is clear from the discussion of published methods above that far more methods have been developed than just the selection listed in Table 2 that are available for installing locally. This suggests that further improvement of MS research may be achieved by wider distribution of some of these methods. An objective evaluation of the performance of those methods should then be a first step. In order to distinguish between disease-related effects (different disease types, patient selection, follow-up durations, etc.) and method-related effects, such comparisons between analysis methods should be performed using the same common dataset(s). A database such as that proposed under recommendation (3) would facilitate such a comparison.

\section{Standard test dataset}

\section{Position statement}

A standard dataset with benchmark results should be set up to facilitate development, calibration, and objective evaluation of image analysis methods for MS. 


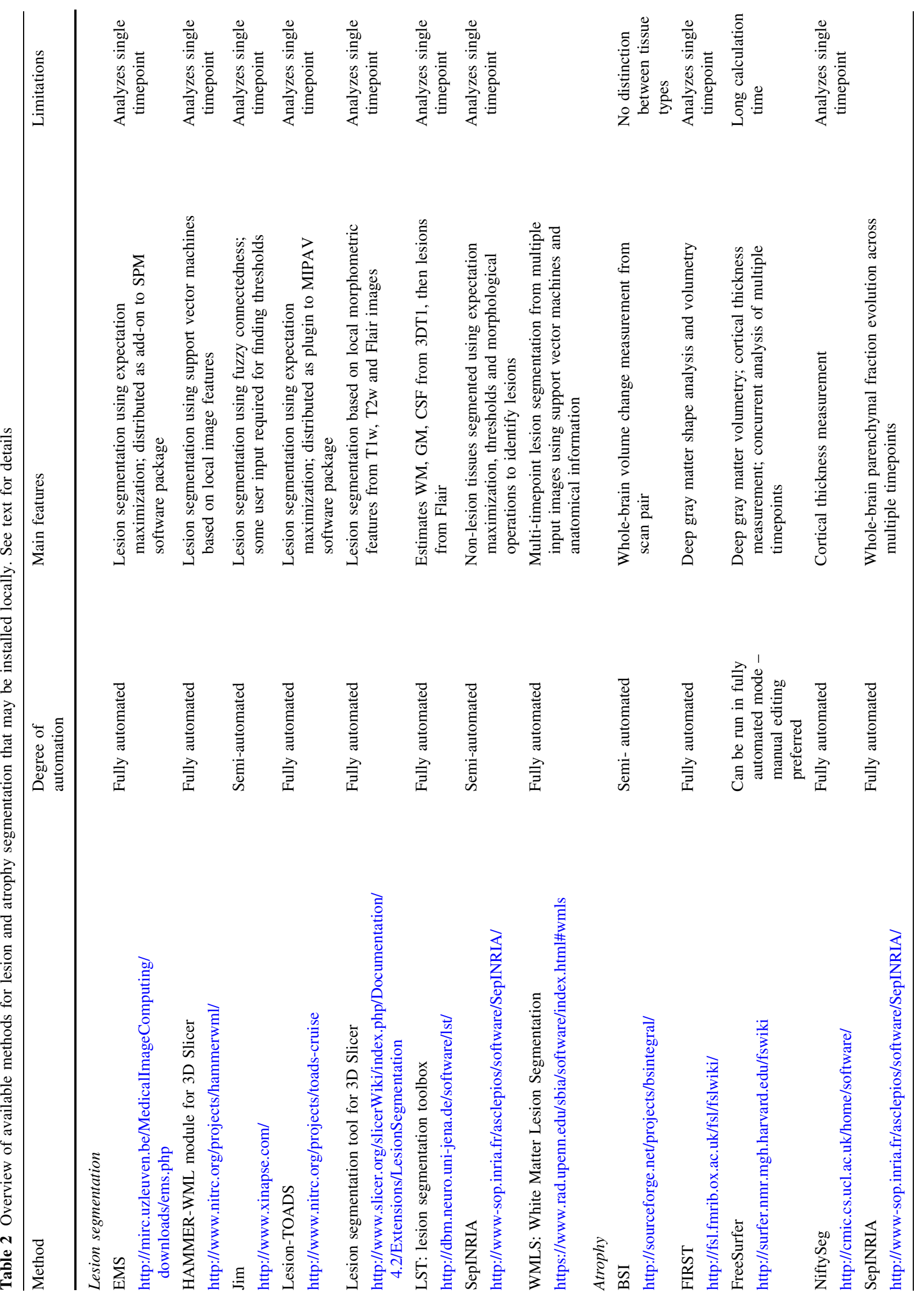




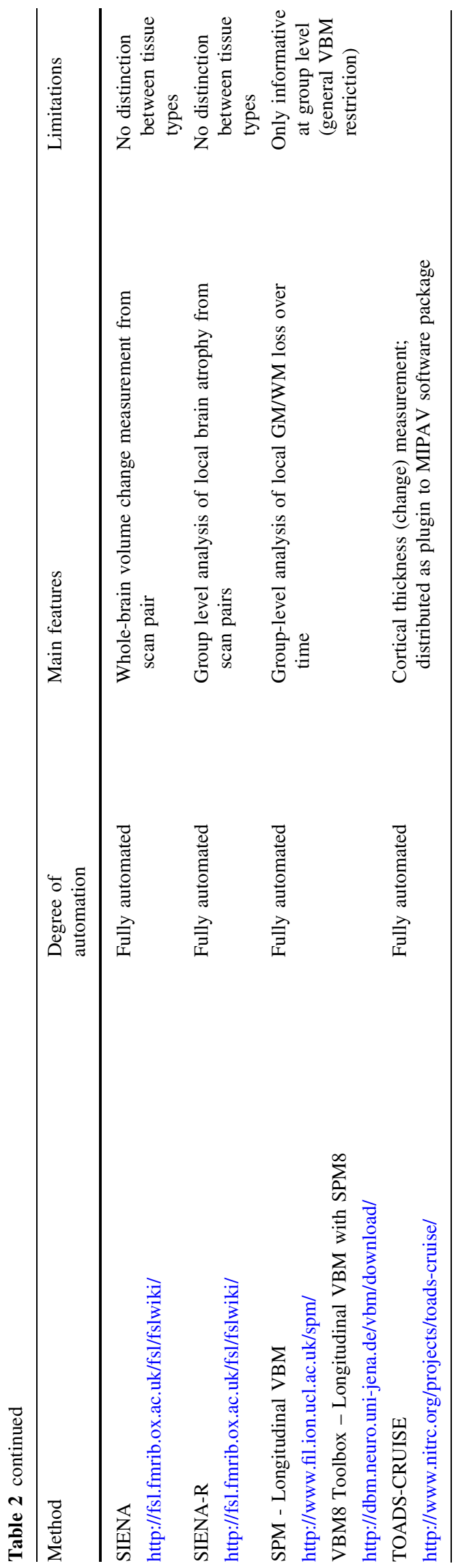

Reasoning

Comparing the performance of one method for lesion load or atrophy measurement to another is difficult due to the lack of standardized representative data. Papers describing new methods do not always compare the new method to current ones, and even if they do, the test image dataset is rarely made available to the larger research community. Finally, different papers use different metrics to report performance of their algorithms. Hence, the results cannot be reproduced in detail, nor can they realistically be compared between methods.

In order to allow investigators to select an analysis method, based on an unbiased assessment of various alternatives, one possible approach is to create annotated longitudinal MR image datasets from carefully selected and representative MS patients from multiple scanners/ centers. This database would consist of different subsets of images for addressing specific questions. The database should be accompanied by the framework necessary for carrying out objective and quantitative evaluation of different methods against "gold standard" expert annotations, including standards for reporting the results of those comparisons, and thereby facilitate an unbiased and transparent assessment of image analysis methods.

Several databases are available that meet some of these requirements. First, BrainWeb (http://www.bic.mni.mcgill. $\mathrm{ca} / \mathrm{brainweb/)} \mathrm{[95]} \mathrm{offers} \mathrm{a} \mathrm{simulated} \mathrm{dataset} \mathrm{in} \mathrm{which,} \mathrm{for}$ a limited number of cases with MS lesions, image characteristics such as intensity spatial inhomogeneity and noise can be varied. Such data could be expanded by including a larger range of lesion volumes and degrees of atrophy in the simulated images. Inserting artificial lesions into images obtained from healthy controls is an approach followed in several papers assessing lesion segmentation or the effect of lesions on atrophy measurement. The advantages of this approach are that the effects of lesions can be studied in isolation, and that the ground truth is known. The main disadvantage is that healthy control images may not be similar to MS patients' images in all respects; for example, the degree of brain atrophy may differ, or "dirty" WM may be present in MS patients while it is generally absent in healthy controls. Therefore, a test dataset should not be restricted to simulated images based on healthy control data, but should also include real patient data.

The image data for the MICCAI 2008 "MS lesion segmentation challenge" do consist of real patient data, derived from a relatively large set of patients; these data are still available online (http://www.ia.unc.edu/MSseg/). The website provides a test-set of images along with expert annotations, so that results of a segmentation method can be compared to the "gold standard" segmentation. The scores obtained using the different methods that have been 
tested are listed on the website, and new entries are still being frequently added. This is a good example of the kind of standardized test dataset that is needed for optimizing analysis methods in MS. However, some characteristics of the imaging data, such as the spatial resolution of the images, are different from those typically used in a clinical trial setting. Furthermore, in addition to the 2D-FLAIR images that form the dataset, different pulse sequence types such as 3D-FLAIR or 2D dual-echo PD/T2 are also needed to test the robustness of lesion segmentation methods, as well as images such as DIR for GM lesion segmentation.

For developing and optimizing atrophy measurement methods, 3D T1-weighted anatomical images using a pulse sequence such as MP-RAGE are required. The ADNI database for $\mathrm{AD}$, mild cognitive impairment and healthy aging may serve as a good example here (http:// adni.loni.ucla.edu/) [96]; it allows researchers to download and use image data, under certain conditions. ADNI has boosted the development of brain image analysis methods [97], thereby also improving MS research. The availability of two consecutively acquired MP-RAGE scans provides an opportunity to study the reproducibility of methods [98], and including similar scan-rescan data in an MS test dataset would be highly desirable. Another example is the OASIS project, which allows researchers to freely download a dataset that contains images of adults across a large age range, including demented and nondemented elderly (http://www.oasis-brains.org/, [99]). The OASIS dataset also contains short-term rescan images for reliability analyses.

Objectively quantifying the performance of lesion segmentation techniques is particularly challenging, since experts do not generally agree completely on which voxels should be considered as part of a lesion [100, 101]. Segmentation of cortical and subcortical GM presents similar problems. Derakshan et al. [9] performed an elegant comparison between six automated methods and six expert segmentations. Their study showed not only how well the automated methods performed compared to the average expert segmentations, but importantly it also highlighted the variability between experts, which should be taken into account in setting up a database. One of the first uses of the proposed database could be to investigate inter-expert variability, and possibly standardize manual outlining methods in order to improve the validation of automated methods for quantifying lesion volume change and atrophy rates in MS.

Finally, beyond providing test datasets, the utility could serve the analysis method development community even better by providing training data sets. The MICCAI MS lesion challenge has been mentioned above, and the sustained availability of those data allows further development of MS lesion segmentation methods. However, there is a real danger that without independent training data, further apparent improvements may not generalize when the methods are applied in new image datasets with different imaging characteristics. Therefore, it seems imperative that to make real progress, training data should be made available that captures the variability that is encountered in a real clinical or trial setting, not only the variability due to inter-patient differences, but also that due to the heterogeneity of scanners and imaging protocols.

\section{Conclusions}

Data collection and analysis methods for longitudinal MR imaging studies of brain lesion load and brain atrophy in MS have proved to be of great value, but can be improved. We propose to (1) acquire images using 3D acquisition techniques with multiple contrasts and near isotropic spatial resolution; (2) integrate the segmentation of lesions and atrophy measurement; and (3) provide a standard test dataset containing both images and expert annotations for objective testing and evaluation of analysis methods. These points should prove complementary: the standard test dataset may facilitate development and improvement of the integrated segmentation techniques, which in turn would benefit from the isotropic spatial resolution of the acquisition.

Acknowledgement F. Barkhof and R.A. van Schijndel have received funding from the European Commission's Seventh Framework Programme (FP7/2007-2013) under grant agreement $n^{\circ} 283562$ (project "neuGRID4you”).

Conflicts of interest H.V. has received funding for collaborative research projects from Pfizer, Novartis and MerckSerono, and speaker honoraria from Novartis. M.J. has received royalties for the licensing of the FSL software package to commercial enterprises (excluding all academic institutions and hospitals) through Isis Innovation (the commercialisation branch of the University of Oxford). E.F. has received consulting fees, speaker honoraria, and/or research funds from Biogen Idec, Genzyme, Novartis, Pfizer/Wyeth, and Teva. A.R. has received funding for travel and speaker honoraria from Bayer Schering and Teva Pharmaceutical. M.H. is Director and stock holder of Xinapse Systems Ltd. F.F. serves on scientific advisory boards for Bayer Schering, Biogen Idec, Genzyme, Merck Serono, Pfizer, Novartis and Teva Pharmaceutical Industries Ltd.; performs reading services for Parexel; serves on the editorial boards of Cerebrovascular Diseases, Multiple Sclerosis, the Polish Journal of Neurology and Neurosurgery, Therapeutic Advances in Neurological Disorders, Stroke, and the Swiss Archives of Neurology and Psychiatry; and has received speaker honoraria and support from Biogen Idec, Bayer Schering, Merck Serono, Novartis, Sanofi-Aventis and Teva Pharmaceutical Industries Ltd. J.P. serves on the Scientific Board for Charcot Foundation, and has performed advisory work for Biogen Idec, Merck Serono Ltd, Bayer Schering Pharma, Novartis Pharmaceuticals UK Ltd, Teva Pharmaceutical Industries Ltd, Gilenya and Ono Pharmaceutical Co Ltd. She receives research support from the MS Society, QIDIS, Merck Serono Ltd, and Bayer Schering Pharma, plus conference expenses from Novartis and Merck Serono Ltd. 
Open Access This article is distributed under the terms of the Creative Commons Attribution License which permits any use, distribution, and reproduction in any medium, provided the original author(s) and the source are credited.

\section{References}

1. Filippi M, Rocca MA, de Stefano N, Enzinger C, Fisher E, Horsfield MA, Inglese M, Pelletier D, Comi G (2011) Magnetic resonance techniques in multiple sclerosis: the present and the future. Arch Neurol 68:1514-1520

2. Filippi M, Rocca MA, Benedict RH, DeLuca J, Geurts JJ, Rombouts SA, Ron M, Comi G (2010) The contribution of MRI in assessing cognitive impairment in multiple sclerosis. Neurology 75:2121-2128

3. Barkhof F, Calabresi PA, Miller DH, Reingold SC (2009) Imaging outcomes for neuroprotection and repair in multiple sclerosis trials. Nat Rev Neurol 5:256-266

4. Barkhof F, Simon JH, Fazekas F, Rovaris M, Kappos L, de Stefano N, Polman CH, Petkau J, Radue EW, Sormani MP, Li DK, O'Connor P, Montalban X, Miller DH, Filippi M (2012) MRI monitoring of immunomodulation in relapse-onset multiple sclerosis trials. Nat Rev Neurol 8:13-21

5. Geurts JJ, Roosendaal SD, Calabrese M, Ciccarelli O, Agosta F, Chard DT, Gass A, Huerga E, Moraal B, Pareto D, Rocca MA, Wattjes MP, Yousry TA, Uitdehaag BM, Barkhof F (2011) Consensus recommendations for MS cortical lesion scoring using double inversion recovery MRI. Neurology 76:418-424

6. Durand-Dubief F, Belaroussi B, Armspach JP, Dufour M, Roggerone S, Vukusics S, Hannoun S, Sappey-Marinier D, Confavreux C, Cotton F (2012) Reliability of longitudinal brain volume loss measurements between 2 sites in patients with multiple sclerosis: comparison of 7 quantification techniques. AJNR Am J Neuroradiol 33(10):1918-1924. doi:10.3174/ajnr. A3107

7. Enzinger C, Fazekas F, Matthews PM, Ropele S, Schmidt H, Smith S, Schmidt R (2005) Risk factors for progression of brain atrophy in aging: six-year follow-up of normal subjects. Neurology 64:1704-1711

8. Miller DH, Barkhof F, Frank JA, Parker GJ, Thompson AJ (2002) Measurement of atrophy in multiple sclerosis: pathological basis, methodological aspects and clinical relevance. Brain 125:1676-1695

9. Derakhshan M, Caramanos Z, Giacomini PS, Narayanan S, Maranzano J, Francis SJ, Arnold DL, Collins DL (2010) Evaluation of automated techniques for the quantification of grey matter atrophy in patients with multiple sclerosis. Neuroimage 52:1261-1267

10. Filippi M, Rocca MA, Barkhof F, Bruck W, Chen JT, Comi G, DeLuca G, de Stefano N, Erickson BJ, Evangelou N, Fazekas F, Geurts JJ, Lucchinetti C, Miller DH, Pelletier D, Popescu BF, Lassmann H (2012) Association between pathological and MRI findings in multiple sclerosis. Lancet Neurol 11:349-360

11. Filippi M, Horsfield MA, Ader HJ, Barkhof F, Bruzzi P, Evans A, Frank JA, Grossman RI, McFarland HF, Molyneux P, Paty DW, Simon J, Tofts PS, Wolinsky JS, Miller DH (1998) Guidelines for using quantitative measures of brain magnetic resonance imaging abnormalities in monitoring the treatment of multiple sclerosis. Ann Neurol 43:499-506

12. Gedamu EL, Gedamu A (2012) Subject movement during multislice interleaved MR acquisitions: prevalence and potential effect on MRI-derived brain pathology measurements and multicenter clinical trials of therapeutics for multiple sclerosis. J Magn Reson Imaging 36:332-343
13. Moraal B, Roosendaal SD, Pouwels PJ, Vrenken H, van Schijndel RA, Meier DS, Guttmann CR, Geurts JJ, Barkhof F (2008) Multi-contrast, isotropic, single-slab 3D MR imaging in multiple sclerosis. Eur Radiol 18:2311-2320

14. Barkhof F, Pouwels PJ, Wattjes MP (2011) The Holy Grail in diagnostic neuroradiology: 3T or 3D? Eur Radiol 21:449-456

15. Mikol DD, Barkhof F, Chang P, Coyle PK, Jeffery DR, Schwid SR, Stubinski B, Uitdehaag BM (2008) Comparison of subcutaneous interferon beta-1a with glatiramer acetate in patients with relapsing multiple sclerosis (the REbif vs Glatiramer Acetate in relapsing MS disease [REGARD] study): a multicentre, randomised, parallel, open-label trial. Lancet Neurol 7:903-914

16. Radue EW, O'Connor P, Polman CH, Hohlfeld R, Calabresi P, Selmaj K, Mueller-Lenke N, Agoropoulou C, Holdbrook F, de Vera A, Zhang-Auberson L, Francis G, Burtin P, Kappos L (2012) Impact of fingolimod therapy on magnetic resonance imaging outcomes in patients with multiple sclerosis. Arch Neurol 1-11. doi:10.1001/archneurol.2012.1051

17. Bagnato F, Butman JA, Gupta S, Calabrese M, Pezawas L, Ohayon JM, Tovar-Moll F, Riva M, Cao MM, Talagala SL, McFarland HF (2006) In vivo detection of cortical plaques by MR imaging in patients with multiple sclerosis. AJNR Am J Neuroradiol 27:2161-2167

18. Bagnato F, Yao B, Cantor F, Merkle H, Condon E, Montequin M, Moore S, Quezado M, Tkaczyk D, McFarland H (2009) Multisequence-imaging protocols to detect cortical lesions of patients with multiple sclerosis: observations from a post-mortem 3 Tesla imaging study. J Neurol Sci 282:80-85

19. de Graaf WL, Zwanenburg JJ, Visser F, Wattjes MP, Pouwels PJ, Geurts JJ, Polman CH, Barkhof F, Luijten PR, Castelijns JA (2012) Lesion detection at seven Tesla in multiple sclerosis using magnetisation prepared 3D-FLAIR and 3D-DIR. Eur Radiol 22:221-231

20. Mike A, Glanz BI, Hildenbrand P, Meier D, Bolden K, Liguori M, Dell'oglio E, Healy BC, Bakshi R, Guttmann CR (2011) Identification and clinical impact of multiple sclerosis cortical lesions as assessed by routine $3 \mathrm{~T}$ MR imaging. AJNR Am J Neuroradiol 32:515-521

21. Simon B, Schmidt S, Lukas C, Gieseke J, Traber F, Knol DL, Willinek WA, Geurts JJ, Schild HH, Barkhof F, Wattjes MP (2010) Improved in vivo detection of cortical lesions in multiple sclerosis using double inversion recovery MR imaging at 3 Tesla. Eur Radiol 20:1675-1683

22. Nelson F, Poonawalla AH, Hou P, Huang F, Wolinsky JS, Narayana PA (2007) Improved identification of intracortical lesions in multiple sclerosis with phase-sensitive inversion recovery in combination with fast double inversion recovery MR imaging. AJNR Am J Neuroradiol 28:1645-1649

23. Sethi V, Yousry TA, Muhlert N, Ron M, Golay X, WheelerKingshott C, Miller DH, Chard DT (2012) Improved detection of cortical MS lesions with phase-sensitive inversion recovery MRI. J Neurol Neurosurg Psychiatry 83:877-882

24. Charil A, Zijdenbos AP, Taylor J, Boelman C, Worsley KJ, Evans AC, Dagher A (2003) Statistical mapping analysis of lesion location and neurological disability in multiple sclerosis: application to 452 patient data sets. Neuroimage 19:532-544

25. Di Perri C, Battaglini M, Stromillo ML, Bartolozzi ML, Guidi L, Federico A, de Stefano N (2008) Voxel-based assessment of differences in damage and distribution of white matter lesions between patients with primary progressive and relapsingremitting multiple sclerosis. Arch Neurol 65:236-243

26. Sepulcre J, Masdeu JC, Sastre-Garriga J, Goni J, Velez-deMendizabal N, Duque B, Pastor MA, Bejarano B, Villoslada P (2008) Mapping the brain pathways of declarative verbal memory: evidence from white matter lesions in the living human brain. Neuroimage 42:1237-1243 
27. Sepulcre J, Masdeu JC, Pastor MA, Goni J, Barbosa C, Bejarano B, Villoslada P (2009) Brain pathways of verbal working memory: a lesion-function correlation study. Neuroimage 47:773-778

28. Vellinga MM, Geurts JJ, Rostrup E, Uitdehaag BM, Polman CH, Barkhof F, Vrenken H (2009) Clinical correlations of brain lesion distribution in multiple sclerosis. J Magn Reson Imaging 29:768-773

29. Kincses ZT, Ropele S, Jenkinson M, Khalil M, Petrovic K, Loitfelder M, Langkammer C, Aspeck E, Wallner-Blazek M, Fuchs S, Jehna M, Schmidt R, Vecsei L, Fazekas F, Enzinger C (2011) Lesion probability mapping to explain clinical deficits and cognitive performance in multiple sclerosis. Mult Scler 17:681-689

30. Bendfeldt K, Blumhagen JO, Egger H, Loetscher P, Denier N, Kuster P, Traud S, Mueller-Lenke N, Naegelin Y, Gass A, Hirsch J, Kappos L, Nichols TE, Radue EW, Borgwardt SJ (2010) Spatiotemporal distribution pattern of white matter lesion volumes and their association with regional grey matter volume reductions in relapsing-remitting multiple sclerosis. Hum Brain Mapp 31:1542-1555

31. Filli L, Hofstetter L, Kuster P, Traud S, Mueller-Lenke N, Naegelin Y, Kappos L, Gass A, Sprenger T, Nichols TE, Vrenken H, Barkhof F, Polman C, Radue EW, Borgwardt SJ, Bendfeldt K (2012) Spatiotemporal distribution of white matter lesions in relapsing-remitting and secondary progressive multiple sclerosis. Mult Scler 18(11):1577-1584. doi:10.1177/ 1352458512442756

32. Moraal B, Wattjes MP, Geurts JJ, Knol DL, van Schijndel RA, Pouwels PJ, Vrenken H, Barkhof F (2010) Improved detection of active multiple sclerosis lesions: 3D subtraction imaging. Radiology 255:154-163

33. Bodini B, Khaleeli Z, Cercignani M, Miller DH, Thompson AJ, Ciccarelli O (2009) Exploring the relationship between white matter and gray matter damage in early primary progressive multiple sclerosis: an in vivo study with TBSS and VBM. Hum Brain Mapp 30:2852-2861

34. Henry RG, Shieh M, Amirbekian B, Chung S, Okuda DT, Pelletier D (2009) Connecting white matter injury and thalamic atrophy in clinically isolated syndromes. J Neurol Sci 282:61-66

35. Zhuo J, Gullapalli RP (2006) AAPM/RSNA physics tutorial for residents: MR artifacts, safety, and quality control. Radiographics 26:275-297

36. Ashburner J, Friston KJ (2005) Unified segmentation. Neuroimage 26:839-851

37. Zhang Y, Brady M, Smith S (2001) Segmentation of brain MR images through a hidden Markov random field model and the expectation-maximization algorithm. IEEE Trans Med Imaging 20:45-57

38. Sled JG, Zijdenbos AP, Evans AC (1998) A nonparametric method for automatic correction of intensity nonuniformity in MRI data. IEEE Trans Med Imaging 17:87-97

39. Caramanos Z, Fonov VS, Francis SJ, Narayanan S, Pike GB, Collins DL, Arnold DL (2010) Gradient distortions in MRI: characterizing and correcting for their effects on SIENA-generated measures of brain volume change. Neuroimage 49:1601-1611

40. Takao H, Abe O, Hayashi N, Kabasawa H, Ohtomo K (2010) Effects of gradient non-linearity correction and intensity nonuniformity correction in longitudinal studies using structural image evaluation using normalization of atrophy (SIENA). J Magn Reson Imaging 32:489-492

41. Jovicich J, Czanner S, Greve D, Haley E, van der KA, Gollub R, Kennedy D, Schmitt F, Brown G, Macfall J, Fischl B, Dale A (2006) Reliability in multi-site structural MRI studies: effects of gradient non-linearity correction on phantom and human data. Neuroimage 30:436-443
42. Smith SM, Zhang Y, Jenkinson M, Chen J, Matthews PM, Federico A, de Stefano N (2002) Accurate, robust, and automated longitudinal and cross-sectional brain change analysis. Neuroimage 17:479-489

43. Battaglini M, Jenkinson M, de Stefano N (2012) Evaluating and reducing the impact of white matter lesions on brain volume measurements. Hum Brain Mapp 33(9):2062-2071. doi: 10.1002/hbm.21344

44. Ceccarelli A, Jackson JS, Tauhid S, Arora A, Gorky J, Dell'oglio E, Bakshi A, Chitnis T, Khoury SJ, Weiner HL, Guttmann CR, Bakshi R, Neema M (2012) The Impact of Lesion InPainting and Registration Methods on Voxel-Based Morphometry in Detecting Regional Cerebral Gray Matter Atrophy in Multiple Sclerosis. AJNR Am J Neuroradiol 33(8):1579-1585. doi:10.3174/ajnr.A3083

45. Chard DT, Jackson JS, Miller DH, Wheeler-Kingshott CA (2010) Reducing the impact of white matter lesions on automated measures of brain gray and white matter volumes. J Magn Reson Imaging 32:223-228

46. Gelineau-Morel R, Tomassini V, Jenkinson M, Johansen-Berg H, Matthews PM, Palace J (2012) The effect of hypointense white matter lesions on automated gray matter segmentation in multiple sclerosis. Hum Brain Mapp 33(12):2802-2814. doi: 10.1002/hbm.21402

47. Nakamura K, Fisher E (2009) Segmentation of brain magnetic resonance images for measurement of gray matter atrophy in multiple sclerosis patients. Neuroimage 44:769-776

48. Sdika M, Pelletier D (2009) Nonrigid registration of multiple sclerosis brain images using lesion inpainting for morphometry or lesion mapping. Hum Brain Mapp 30:1060-1067

49. Shiee N, Bazin PL, Ozturk A, Reich DS, Calabresi PA, Pham DL (2010) A topology-preserving approach to the segmentation of brain images with multiple sclerosis lesions. Neuroimage 49:1524-1535

50. Nakamura K, Fox R, Fisher E (2011) CLADA: cortical longitudinal atrophy detection algorithm. Neuroimage 54:278-289

51. Reuter M, Schmansky NJ, Rosas HD, Fischl B (2012) Withinsubject template estimation for unbiased longitudinal image analysis. Neuroimage 61:1402-1418

52. Duan Y, Hildenbrand PG, Sampat MP, Tate DF, Csapo I, Moraal B, Bakshi R, Barkhof F, Meier DS, Guttmann CR (2008) Segmentation of subtraction images for the measurement of lesion change in multiple sclerosis. AJNR Am J Neuroradiol 29:340-346

53. Segonne F, Dale AM, Busa E, Glessner M, Salat D, Hahn HK, Fischl B (2004) A hybrid approach to the skull stripping problem in MRI. Neuroimage 22:1060-1075

54. Smith SM (2002) Fast robust automated brain extraction. Hum Brain Mapp 17:143-155

55. Crinion J, Ashburner J, Leff A, Brett M, Price C, Friston K (2007) Spatial normalization of lesioned brains: performance evaluation and impact on fMRI analyses. Neuroimage 37:866-875

56. Jasperse B, Valsasina P, Neacsu V, Knol DL, de Stefano N, Enzinger C, Smith SM, Ropele S, Korteweg T, Giorgio A, Anderson V, Polman CH, Filippi M, Miller DH, Rovaris M, Barkhof F, Vrenken H (2007) Intercenter agreement of brain atrophy measurement in multiple sclerosis patients using manually edited SIENA and SIENAX. J Magn Reson Imaging 26:881-885

57. Popescu V, Battaglini M, Hoogstrate WS, Verfaillie SC, Sluimer IC, van Schijndel RA, van Dijk BW, Cover KS, Knol DL, Jenkinson M, Barkhof F, de Stefano N, Vrenken H (2012) Optimizing parameter choice for FSL-brain extraction tool (BET) on 3D T1 images in multiple sclerosis. Neuroimage 61:1484-1494 
58. Mortazavi D, Kouzani AZ, Soltanian-Zadeh H (2012) Segmentation of multiple sclerosis lesions in MR images: a review. Neuroradiology 54:299-320

59. Goldberg-Zimring D, Achiron A, Miron S, Faibel M, Azhari H (1998) Automated detection and characterization of multiple sclerosis lesions in brain MR images. Magn Reson Imaging $16: 311-318$

60. Hojjatoleslami SA, Kruggel F, von Cramon DY (1999) Segmentation of white matter lesions from volumetric MR images. In: Proceedings on Medical image computing and computerassisted intervention, Miccai'99, 1999, 1679: 52-61

61. Pachai C, Zhu YM, Grimaud J, Hermier M, Dromigny-Badin A, Boudraa A, Gimenez G, Confavreux C, Froment JC (1998) A pyramidal approach for automatic segmentation of multiple sclerosis lesions in brain MRI. Comput Med Imaging Graph 22:399-408

62. Khayati R, Vafadust M, Towhidkhah F, Nabavi M (2008) Fully automatic segmentation of multiple sclerosis lesions in brain MR FLAIR images using adaptive mixtures method and Markov random field model. Comput Biol Med 38:379-390

63. Freifeld O, Greenspan H, Goldberger J (2009) Multiple sclerosis lesion detection using constrained GMM and curve evolution. Int J Biomed Imaging 2009:715124

64. Anbeek P, Vincken KL, van Osch MJ, Bisschops RH, van der Grond J (2004) Probabilistic segmentation of white matter lesions in MR imaging. Neuroimage 21:1037-1044

65. Anbeek P, Vincken KL, van Osch MJ, Bisschops RH, van der Grond J (2004) Automatic segmentation of different-sized white matter lesions by voxel probability estimation. Med Image Anal $8: 205-215$

66. Mohamed FB, Vinitski S, Gonzalez CF, Faro SH, Lublin FA, Knobler R, Gutierrez JE (2001) Increased differentiation of intracranial white matter lesions by multispectral 3D-tissue segmentation: preliminary results. Magn Reson Imaging 19:207-218

67. Horsfield MA, Bakshi R, Rovaris M, Rocca MA, Dandamudi VS, Valsasina P, Judica E, Lucchini F, Guttmann CR, Sormani MP, Filippi M (2007) Incorporating domain knowledge into the fuzzy connectedness framework: application to brain lesion volume estimation in multiple sclerosis. IEEE Trans Med Imaging 26:1670-1680

68. Udupa JK, Wei L, Samarasekera S, Miki Y, van Buchem MA, Grossman RI (1997) Multiple sclerosis lesion quantification using fuzzy-connectedness principles. IEEE Trans Med Imaging 16:598-609

69. van Leemput K, Maes F, Vandermeulen D, Colchester A, Suetens P (2001) Automated segmentation of multiple sclerosis lesions by model outlier detection. IEEE Trans Med Imaging 20:677-688

70. Lao Z, Shen D, Liu D, Jawad AF, Melhem ER, Launer LJ, Bryan RN, Davatzikos C (2008) Computer-assisted segmentation of white matter lesions in 3D MR images using support vector machine. Acad Radiol 15:300-313

71. Zijdenbos AP, Forghani R, Evans AC (2002) Automatic "pipeline" analysis of 3D MRI data for clinical trials: application to multiple sclerosis. IEEE Trans Med Imaging 21:1280-1291

72. Molyneux PD, Wang L, Lai M, J G, Tofts PS, Moseley IF, Miller DH (1998) Quantitative techniques for lesion load measurement in multiple sclerosis: an assessment of the global threshold technique after non uniformity and histogram matching corrections. Eur J Neurol 5:55-60

73. Wang L, Lai HM, Barker GJ, Miller DH, Tofts PS (1998) Correction for variations in MRI scanner sensitivity in brain studies with histogram matching. Magn Reson Med 39:322-327

74. Wicks DA, Tofts PS, Miller DH, du Boulay GH, Feinstein A, Sacares RP, Harvey I, Brenner R, McDonald WI (1992) Volume measurement of multiple sclerosis lesions with magnetic resonance images. A preliminary study. Neuroradiology 34:475-479

75. Grimaud J, Lai M, Thorpe J, Adeleine P, Wang L, Barker GJ, Plummer DL, Tofts PS, McDonald WI, Miller DH (1996) Quantification of MRI lesion load in multiple sclerosis: a comparison of three computer-assisted techniques. Magn Reson Imaging 14:495-505

76. Molyneux PD, Tofts PS, Fletcher A, Gunn B, Robinson P, Gallagher H, Moseley IF, Barker GJ, Miller DH (1998) Precision and reliability for measurement of change in MRI lesion volume in multiple sclerosis: a comparison of two computer assisted techniques. J Neurol Neurosurg Psychiatry 65:42-47

77. Gawne-Cain ML, O'Riordan JI, Coles A, Newell B, Thompson AJ, Miller DH (1998) MRI lesion volume measurement in multiple sclerosis and its correlation with disability: a comparison of fast fluid attenuated inversion recovery (fFLAIR) and spin echo sequences. J Neurol Neurosurg Psychiatry 64:197-203

78. Llado X, Ganiler O, Oliver A, Marti R, Freixenet J, Valls L, Vilanova JC, Ramio-Torrenta L, Rovira A (2012) Automated detection of multiple sclerosis lesions in serial brain MRI. Neuroradiology 54:787-807

79. Moraal B, Meier DS, Poppe PA, Geurts JJ, Vrenken H, Jonker WM, Knol DL, van Schijndel RA, Pouwels PJ, Pohl C, Bauer L, Sandbrink R, Guttmann CR, Barkhof F (2009) Subtraction MR images in a multiple sclerosis multicenter clinical trial setting. Radiology 250:506-514

80. Moraal B, van den Elskamp I, Knol DL, Uitdehaag BM, Geurts JJ, Vrenken H, van Schijndel RA, Meier DS, Guttmann CR (2010) Long-interval T2-weighted subtraction magnetic resonance imaging: a powerful new outcome measure in multiple sclerosis trials. Ann Neurol 67:667-675

81. Ait-Ali LS, Prima S, Hellier P, Carsin B, Edan G, Barillot C (2005) STREM: A robust multidimensional parametric method to segment MS lesions in MRI. In: Proceedings on medical image computing and computer-assisted intervention-Miccai 2005, Pt 1 3749:409-416

82. Gerig G, Welti D, Guttmann CR, Colchester AC, Szekely G (2000) Exploring the discrimination power of the time domain for segmentation and characterization of active lesions in serial MR data. Med Image Anal 4:31-42

83. Rey D, Subsol G, Delingette H, Ayache N (2002) Automatic detection and segmentation of evolving processes in $3 \mathrm{D}$ medical images: application to multiple sclerosis. Med Image Anal 6:163-179

84. Thirion JP, Calmon G (1999) Deformation analysis to detect and quantify active lesions in three-dimensional medical image sequences. IEEE Trans Med Imaging 18:429-441

85. Pieperhoff P, Sudmeyer M, Homke L, Zilles K, Schnitzler A, Amunts K (2008) Detection of structural changes of the human brain in longitudinally acquired MR images by deformation field morphometry: methodological analysis, validation and application. Neuroimage 43:269-287

86. Giorgio A, Battaglini M, Smith SM, de Stefano N (2008) Brain atrophy assessment in multiple sclerosis: importance and limitations. Neuroimaging Clin N Am 18:675-686 xi

87. Kezele IB, Chen JT, Arnold DL, Collins DL (2007) The relation of focal white matter signal abnormality and focal volume loss in multiple sclerosis. Mult Scler 13:809-813

88. Freeborough PA, Fox NC (1997) The boundary shift integral: an accurate and robust measure of cerebral volume changes from registered repeat MRI. IEEE Trans Med Imaging $16: 623-629$

89. Smith SM, Rao A, de Stefano N, Jenkinson M, Schott JM, Matthews PM, Fox NC (2007) Longitudinal and cross-sectional analysis of atrophy in Alzheimer's disease: cross-validation of BSI. SIENA and SIENAX. Neuroimage 36:1200-1206 
90. Anderson VM, Bartlett JW, Fox NC, Fisniku L, Miller DH (2007) Detecting treatment effects on brain atrophy in relapsing remitting multiple sclerosis: sample size estimates. J Neurol 254:1588-1594

91. Camara O, Schnabel JA, Ridgway GR, Crum WR, Douiri A, Scahill RI, Hill DL, Fox NC (2008) Accuracy assessment of global and local atrophy measurement techniques with realistic simulated longitudinal Alzheimer's disease images. Neuroimage 42:696-709

92. Battaglini M, Giorgio A, Stromillo ML, Bartolozzi ML, Guidi L, Federico A, de Stefano N (2009) Voxel-wise assessment of progression of regional brain atrophy in relapsing-remitting multiple sclerosis. J Neurol Sci 282:55-60

93. Takao H, Hayashi N, Ohtomo K (2011) Effect of scanner in longitudinal studies of brain volume changes. J Magn Reson Imaging 34:438-444

94. Bendfeldt K, Hofstetter L, Kuster P, Traud S, Mueller-Lenke N, Naegelin Y, Kappos L, Gass A, Nichols TE, Barkhof F, Vrenken H, Roosendaal SD, Geurts JJ, Radue EW, Borgwardt SJ (2012) Longitudinal gray matter changes in multiple sclerosis-differential scanner and overall disease-related effects. Hum Brain Mapp 33:1225-1245

95. Collins DL, Zijdenbos AP, Kollokian V, Sled JG, Kabani NJ, Holmes CJ, Evans AC (1998) Design and construction of a realistic digital brain phantom. IEEE Trans Med Imaging 17:463-468

96. Jack CR Jr, Bernstein MA, Fox NC, Thompson P, Alexander G, Harvey D, Borowski B, Britson PJ, Whitwell L, Ward C, Dale AM, Felmlee JP, Gunter JL, Hill DL, Killiany R, Schuff N, FoxBosetti S, Lin C, Studholme C, DeCarli CS, Krueger G, Ward HA, Metzger GJ, Scott KT, Mallozzi R, Blezek D, Levy J,
Debbins JP, Fleisher AS, Albert M, Green R, Bartzokis G, Glover G, Mugler J, Weiner MW (2008) The Alzheimer's disease neuroimaging initiative (ADNI): MRI methods. J Magn Reson Imaging 27:685-691

97. Weiner MW, Veitch DP, Aisen PS, Beckett LA, Cairns NJ, Green RC, Harvey D, Jack CR, Jagust W, Liu E, Morris JC, Petersen RC, Saykin AJ, Schmidt ME, Shaw L, Siuciak JA, Soares H, Toga AW, Trojanowski JQ (2012) The Alzheimer's disease neuroimaging initiative: a review of papers published since its inception. Alzheimers Dement 8:S1-68

98. Cover KS, van Schijndel RA, van Dijk BW, Redolfi A, Knol DL, Frisoni GB, Barkhof F, Vrenken H, neuGRID, Alzheimer's Disease Neuroimaging Initiative (2011) Assessing the reproducibility of the SienaX and Siena brain atrophy measures using the ADNI back-to-back MP-RAGE MRI scans. Psychiatry Res 193:182-190

99. Marcus DS, Wang TH, Parker J, Csernansky JG, Morris JC, Buckner RL (2007) Open access series of imaging studies (OASIS): cross-sectional MRI data in young, middle aged, non demented, and demented older adults. J Cogn Neurosci 19:1498-1507

100. Filippi M, Horsfield MA, Bressi S, Martinelli V, Baratti C, Reganati P, Campi A, Miller DH, Comi G (1995) Intra- and inter-observer agreement of brain MRI lesion volume measurements in multiple sclerosis. A comparison of techniques. Brain 118(Pt 6):1593-1600

101. Filippi M, Rovaris M, Sormani MP, Horsfield MA, Rocca MA, Capra R, Prandini F, Comi G (1998) Intraobserver and interobserver variability in measuring changes in lesion volume on serial brain MR images in multiple sclerosis. AJNR Am J Neuroradiol 19:685-687 